\title{
Continued fraction solution for the radiative transfer equation in three dimensions
}

\author{
Marián Boguñá, ${ }^{1, *}$ Josep M. Porrà, ${ }^{2, \dagger}$ and Jaume Masoliver ${ }^{2}$ \\ ${ }^{1}$ Center for Information Technology, National Institutes of Health, Bethesda, Maryland 20892 \\ ${ }^{2}$ Departament de Física Fonamental, Universitat de Barcelona, Diagonal 647, 08028 Barcelona, Spain
}

(Received 1 February 2000)

\begin{abstract}
Starting from the radiative transfer equation, we obtain an analytical solution for both the free propagator along one of the axes and an arbitrary phase function in the Fourier-Laplace domain. We also find the effective absorption parameter, which turns out to be very different from the one provided by the diffusion approximation. We finally present an analytical approximation procedure and obtain a differential equation that accurately reproduces the transport process. We test our approximations by means of simulations that use the HenyeyGreenstein phase function with very satisfactory results.

PACS number(s): 05.40.-a, 05.60.-k, 66.90.+r
\end{abstract}

\section{INTRODUCTION}

Diffusion theory is perhaps the most employed approximation scheme for photon migration in multiply scattering media because its simplicity goes along with effectivity in many applications [1-4]. There are, of course, situations where diffusion theory does not provide useful approximations and simply does not work. One of these cases is that of transport in either small systems or through strong absorbing media. In other words, diffusion theory will not provide correct approximations when the photon mean lifetime inside the medium is small compared to the so-called isotropization time $[5,6]$, since in such a case photons do not experience enough collisions for the diffusion approximation to apply [4]. This is certainly the case of transport through thin slabs, the usual geometry encountered in many biological experiments such as skin measurements and nondestructive tests [2].

One of the reasons for the failure of diffusion theory in thin slabs is that it does not take into account the ballistic properties of photon movements and the effect of anisotropic scattering. Several approaches have been developed to overcome these difficulties; among them we single out the telegrapher's equation approach first proposed by Ishimaru [7] some years ago and recently improved by Durian and Rudnick by means of an ad hoc telegrapher's equation adapted to the problem [8]. Another approach, proposed by Gandjbakhche, Bonner, and Nossal $[5,6]$, exploits the random-walk image of multiple light scattering with properly scaled parameters, so as to take anisotropy into account. We have recently addressed the problem as well and proposed a model based on a three-dimensional generalization of the persistent random walk [9]. This model assumes that photons move along directions that are parallel to the axes, which allows taking ballistic motion effects and strongly anisotropic scattering into account. However, the model is unrealistic in spite of allowing us to exactly solve the problem of multiplescattering migration in a thin slab.

\footnotetext{
*Electronic address: bogunya@helix.nih.gov

${ }^{\dagger}$ Present address: Gaesco Bolsa, SVB, S.A., Diagonal 429, 08036

Barcelona, Spain.
}

None of the above approximations are completely satisfactory, and the most complete account of transport for the problem at hand is provided only by the solution of the transport equation, which, in the case of light propagation through narrow slabs, is the so-called radiative transfer equation (see below). Nevertheless, to our knowledge, there are no general analytical solutions other than numerical ones for transport equations, and this makes fitting experimental data to theory difficult and impractical. Herein we obtain an analytical solution, for the free propagator along one axis and for any phase function, of the radiative transfer equation in the Fourier-Laplace domain. We also present an approximation scheme, based on continued fractions, which gives very good results when tested against simulation results.

\section{MATHEMATICAL FRAMEWORK}

The starting point of our analysis is the radiative transfer equation (RTE) that defines the motion of a photon through a disordered medium $[1,7,10]$. The photon moves through the medium at finite velocity $c$ and suffers scattering according to a Poisson law at rate $\mu_{s}$; that is, the time between two consecutive scattering events is a random variable with probability density

$$
\psi(t)=\mu_{s} e^{-\mu_{s} t} .
$$

These scattering events are defined by a phase function $\beta\left(\boldsymbol{\Omega} \mid \boldsymbol{\Omega}^{\prime}\right)$ that takes into account the probability of a given transition between two directions of propagation, $\boldsymbol{\Omega}^{\prime}$ and $\boldsymbol{\Omega}$. Moreover, we will assume that the phase function is only a function of the relative angle between the directions before and after the collision. This is not a strong assumption as long as no drift is acting on the system, as is the case of photon propagation in homogeneous media. This implies that the phase function takes the simpler form

$$
\beta\left(\boldsymbol{\Omega} \mid \boldsymbol{\Omega}^{\prime}\right)=\frac{1}{2 \pi} \beta(\theta),
$$

where $\theta$ is the relative angle; that is, $\cos \theta=\boldsymbol{\Omega} \cdot \boldsymbol{\Omega}^{\prime}$. Finally, let $p(\boldsymbol{r}, \boldsymbol{\Omega}, t)$ be the joint probability density function in order 
to find the photon at the position $\boldsymbol{r}=(x, y, z)$ moving in the direction $\boldsymbol{\Omega}$ at time $t$. The radiative transfer equation for this density is [10]

$$
\begin{gathered}
\frac{\partial p(\boldsymbol{r}, \boldsymbol{\Omega}, t)}{\partial t}+c \boldsymbol{\Omega} \cdot \boldsymbol{\nabla} p(\boldsymbol{r}, \boldsymbol{\Omega}, t)+\mu_{s} p(\boldsymbol{r}, \boldsymbol{\Omega}, t) \\
=\mu_{s} \int \beta\left(\boldsymbol{\Omega} \mid \boldsymbol{\Omega}^{\prime}\right) p\left(\boldsymbol{r}, \boldsymbol{\Omega}^{\prime}, t\right) d \boldsymbol{\Omega}^{\prime} .
\end{gathered}
$$

One of the most important applications of this equation corresponds to the problem of transport through slabs. In this case, because of the symmetry of the problem, the only relevant direction is one perpendicular to the slab, say, the $z$ axis. Having this in mind, we will solve the problem for the marginal probability density $p(z, t)$. In consequence, we will replace the operator $\boldsymbol{\Omega} \cdot \boldsymbol{\nabla}$ in Eq. (1) by $\cos \theta \partial / \partial z$ and use the initial condition

$$
p(z, \boldsymbol{\Omega}, t=0)=\beta_{0}(\mathbf{\Omega}) \delta(z),
$$

which corresponds to a photon starting its movement from the origin at $t=0$ with direction distributed according to some function $\beta_{0}(\boldsymbol{\Omega})$. Moreover, we will assume that photons start moving with cylindrical symmetry with respect to the $z$ axis; that is, $\beta_{0}(\boldsymbol{\Omega})$ is only a function of the polar angle with respect to the $z$ axis. The problem given by Eqs. (1) and (2) contains all the information we need, and obtaining its solution is the main objective of this paper.

\section{A. The angular expansion and the $\boldsymbol{P}_{N}$ method}

We will now perform the angular expansion on all the functions appearing in the RTE. In fact, this is the basic assumption of the so-called $P_{N}$ approximations that are extensively used in transport theory [10]. Thus the phase function becomes

$$
\beta(\boldsymbol{\Omega} \mid \boldsymbol{\Omega})^{\prime}=\sum_{l=0}^{\infty} \sum_{m=-l}^{l} \beta_{l} \mathcal{Y}_{l m}(\boldsymbol{\Omega}) \mathcal{Y}_{l m}^{*}\left(\mathbf{\Omega}^{\prime}\right)
$$

(symbol * means complex conjugate), where $\mathcal{Y}_{l m}(\mathbf{\Omega})$ are the spherical harmonics defined as

$$
\mathcal{Y}_{l m}(\boldsymbol{\Omega})=\sqrt{\frac{2 l+1}{4 \pi} \frac{(l-m) !}{(l+m) !}} e^{i m \varphi} P_{l}^{m}(\cos \theta),
$$

$P_{l}^{m}(\cdot)$ are the associated Legendre functions, and $\beta_{l}$ are the coefficients of the expansion of the phase function in terms of Legendre polynomials, i.e.,

$$
\beta_{l}=\int_{0}^{\pi} \beta(\theta) P_{l}(\cos \theta) \sin \theta d \theta .
$$

We easily see that $\beta_{0}=1$, which assures the normalization condition, and

$$
\beta_{1}=g \equiv\langle\cos \theta\rangle
$$

is the anisotropic parameter widely used in the literature. Taking into account Eq. (3), the term on the right-hand side of Eq. (1) becomes

$$
\mu_{s} \sum_{l=0}^{\infty} \sum_{m=-l}^{l} \beta_{l} p_{l m}(z, t) \mathcal{Y}_{l m}(\mathbf{\Omega})
$$

where $p_{l m}(z, t)$ are the coefficients in the expansion of $p(z, \boldsymbol{\Omega}, t)$ in terms of spherical harmonics, i.e.,

$$
p_{l m}(z, t)=\int p(z, \boldsymbol{\Omega}, t) \mathcal{Y}_{l m}^{*}(\boldsymbol{\Omega}) d \mathbf{\Omega} .
$$

Collecting all of the above and taking the joint FourierLaplace transform of Eq. (1), we get

$$
\begin{aligned}
\sum_{l=0}^{\infty} \sqrt{\frac{1+2 l}{4 \pi}} \beta_{l}^{0} \mathcal{Y}_{l 0}(\boldsymbol{\Omega}) \\
=\sum_{l=0}^{\infty} \sum_{m=-l}^{l}\left[s+\mu_{s}\left(1-\beta_{l}\right)\right] \hat{p}_{l m}(\omega, s) \mathcal{Y}_{l m}(\mathbf{\Omega}) \\
+i c \omega \sum_{l=0}^{\infty} \sum_{m=-l}^{l} \hat{p}_{l m}(\omega, s) \cos \theta \mathcal{Y}_{l m}(\mathbf{\Omega}),
\end{aligned}
$$

where

$$
\hat{p}_{l m}(\omega, s)=\int_{-\infty}^{\infty} d z e^{-i \omega z} \int_{0}^{\infty} d t e^{-s t} p_{l m}(z, t)
$$

is the joint Fourier-Laplace transform of the coefficients defined in Eq. (5). The quantities $\beta_{l}^{0}$ are defined as in Eq. (4) but with the initial angle distribution $\beta_{0}(\boldsymbol{\Omega})$ instead of $\beta(\boldsymbol{\Omega})$. Using the standard relation

$$
(2 l+1) x P_{l}^{m}(x)=(l+1-m) P_{l+1}^{m}(x)+(l+m) P_{l-1}^{m}(x),
$$

we can obtain from Eq. (6) the following infinite set of linear equations for $\hat{p}_{l 0}$ :

$$
s \hat{p}_{0}(\omega, s)+\frac{i c \omega}{\sqrt{3}} \hat{p}_{1}(\omega, s)=\frac{1}{\sqrt{4 \pi}},
$$

$$
\hat{p}_{l-1}(\omega, s)+\sigma_{l} \hat{p}_{l+1}(\omega, s)=\alpha_{l}(\omega, s) \hat{p}_{l}(\omega, s)+\rho_{l}(\omega)
$$

$(l=1,2,3, \ldots)$ where $\hat{p}_{l}(\omega, s) \equiv \hat{p}_{l 0}(\omega, s)$,

$$
\begin{gathered}
\sigma_{l}=\frac{l+1}{l} \sqrt{\frac{2 l-1}{2 l+3}}, \\
\alpha_{l}(\omega, s)=\frac{i\left[s+\mu_{s}\left(1-\beta_{l}\right)\right] \sqrt{4 l^{2}-1}}{c \omega l},
\end{gathered}
$$

and

$$
\rho_{l}(\omega)=-\frac{2 l+1}{i c \omega l} \sqrt{\frac{2 l-1}{4 \pi}} \beta_{l}^{0} .
$$

Note that we are solving the problem for $\hat{p}_{l 0}(z, t)$ because we are only interested in the marginal probability density $p(z, t)$, which is related to $p_{00}(z, t)$ through the relation $p(z, t)$ $=\sqrt{4 \pi} p_{00}(z, t)$. 
We will solve the system of equations (7) in two steps: (i) we first truncate the infinite set; i.e, we assume that both $\hat{p}_{l}(\omega, s)$ and $\rho_{l}(\omega)$ vanish when $l \geqslant n$ for certain $n$, and solve the resulting finite system of equations; (ii) we take the limit $n \rightarrow \infty$. Following this way, the final solution reads

$$
\begin{aligned}
\hat{p}(\omega, s)= & \frac{1}{s\left[s+\mu_{s}(1-g)\right]+c^{2} \omega^{2} \mathcal{F}_{1}(\omega, s) / 3}\left\{s+\mu_{s}(1-g)\right. \\
& -i c \omega \mathcal{F}_{1}(\omega, s)\left[\beta_{1}^{0}+\sum_{l=1}^{\infty} \frac{(l+1) ! \beta_{l+1}^{0}}{(2 l+1) ! !}\right. \\
& \left.\left.\times(-i c \omega)^{l} \prod_{j=2} \frac{\mathcal{F}_{j}(\omega, s)}{s+\mu_{s}\left(1-\beta_{l}\right)}\right]\right\},
\end{aligned}
$$

where $\mathcal{F}_{j}(\omega, s)$ are continued fractions defined as

$$
\mathcal{F}_{j}(\omega, s)=\frac{1}{1+\frac{\kappa_{j}(\omega, s)}{1+\frac{\kappa_{j+1}(\omega, s)}{1+\frac{\kappa_{j+2}(\omega, s)}{1+\cdots}}}}
$$

and $\kappa_{l}(\omega, s)$ are the following coefficients that contain all the information about the characteristics of the scattering:

$$
\begin{aligned}
& \kappa_{l}(\omega, s) \\
& =\frac{(l+1)^{2} c^{2} \omega^{2}}{(2 l+1)(2 l+3)\left[s+\mu_{s}\left(1-\beta_{l}\right)\right]\left[s+\mu_{s}\left(1-\beta_{l+1}\right)\right]} .
\end{aligned}
$$

Equations (8)-(10) are the main results of the paper and provide us with the exact solution in the Fourier-Laplace space of the marginal probability density $p(z, t)$ for an arbitrary phase function. If we assume that $\kappa_{l}(\omega, s)=0$ for $l$ $\geqslant N$, we recover the so-called $P_{N}$ approximation and, from this point of view, our solution is precisely the $P_{\infty}$ solution.

In order to proceed further, we need to specify a phase function for the problem at hand. The simplest choice is that of the isotropic scattering. In such a case, all coefficients of the phase function vanish; that is, $\beta_{l}=0(l=1,2, \ldots)$ and

$$
\kappa_{l}(\omega, s)=\frac{(l+1)^{2} c^{2} \omega^{2}}{(2 l+1)(2 l+3)\left(s+\mu_{s}\right)^{2}} .
$$

Moreover, if we suppose isotropic initial conditions, then solution (8) becomes

$$
\hat{p}(\omega, s)=\frac{\arctan \left(\frac{c \omega}{s+\mu_{s}}\right)}{c \omega-\mu_{s} \arctan \left(\frac{c \omega}{s+\mu_{s}}\right)},
$$

where we have used the expression of the function $\arctan (x)$ in terms of a continued fraction [13]. In this case, due to the spherical symmetry of the solution, we can replace $\omega$ with $\boldsymbol{\omega}=\sqrt{\omega_{x}^{2}+\omega_{y}^{2}+\omega_{z}^{2}}$ and get the three-dimensional density $\hat{p}(\boldsymbol{\omega}, s)$. This is precisely the solution obtained by Masoliver,
Porrà, and Weiss some years ago [11] and, in a different context, by Claes and Van den Broeck [14].

\section{B. The moments of the distribution}

Let us now obtain the moments of the distribution using the characteristic function Eq. (8). Looking at the structure of the continued fraction (9), we realize that the $\mathcal{F}_{j}(\omega, s)$ are analytical functions of $\omega$ and that their expansion until order $2 n$ coincides with the expansion of the same fraction truncated at $\kappa_{j+l}(\omega, s)=0$ for $l>n$. Using this property, we can easily write the Laplace transform of the moments $\left\langle\hat{z}^{n}(s)\right\rangle$. Thus, for instance, the first three moments read

$$
\langle\hat{z}(s)\rangle=\frac{c \beta_{1}^{0}}{s s_{1}},
$$

$$
\begin{gathered}
\left\langle\hat{z}^{2}(s)\right\rangle=\frac{2 c^{2}}{3 s^{2} s_{1}}+\frac{4 c^{2} \beta_{2}^{0}}{3 s s_{1} s_{2}}, \\
\left\langle\hat{z}^{3}(s)\right\rangle=\frac{2 c^{3} \beta_{1}^{0}}{s^{2} s_{1}^{2}}+\frac{24 c^{3} \beta_{1}^{0}}{15 s s_{1}^{2} s_{2}}+\frac{36 \beta_{3}^{0} c^{3}}{15 s s_{1} s_{2} s_{3}},
\end{gathered}
$$

where, for simplicity of notation, we have defined

$$
s_{n} \equiv s+\mu_{s}\left(1-\beta_{n}\right)
$$

$(n=1,2,3, \ldots)$. From these expressions we readily see that in order to obtain the moment of order $n$ we need to know the $n$th coefficient of the expansion of the phase function. Hence any approximation trying to go further than the diffusion approximation necessarily needs at least the secondorder properties of the phase function.

\section{The differential equation}

In spite of having the exact expression, in terms of the continuous fractions and the Fourier-Laplace domain, of the marginal probability density, it turns out to be quite useful to obtain the differential equation for $p(z, t)$ because the knowledge of this equation will allow us to get suitable approximate expressions of the distribution. Note first that, as mentioned above, the continued fractions of Eq. (9) are analytical functions of $\omega$ that can be expanded in the form

$$
\mathcal{F}_{j}(\omega, s)=1+\sum_{n=1}^{\infty} \hat{f}_{n}^{(j)}(s) \omega^{2 n},
$$

where the coefficients $\hat{f}_{m}^{(j)}(s)$ can be obtained by expanding the equivalent truncated fraction (i.e., with $\kappa_{l}=0$ for $l>n$ ) in powers of $\omega$. Substituting Eq. (15) into Eq. (8) and inverting the joint Fourier-Laplace transform we get 


$$
\begin{aligned}
& \frac{\partial^{2} p(z, t)}{\partial t^{2}}+\mu_{s}(1-g) \frac{\partial p(z, t)}{\partial t} \\
& =\frac{1}{3} c^{2} \frac{\partial^{2} p(z, t)}{\partial z^{2}}+\frac{1}{3} c^{2} \sum_{n=1}^{\infty}(-1)^{n} \\
& \quad \times \int_{0}^{t} f_{n}(t-\tau) \frac{\partial^{2(n+1)} p(z, \tau)}{\partial z^{2(m+1)}} d \tau+g(z, t),
\end{aligned}
$$

where $f_{n}(t) \equiv f_{n}^{(1)}(t)$. The inhomogeneous term $g(z, t)$ contains the information about the second-order properties of the initial conditions; that is, it contains the coefficients $\beta_{l}^{0}$ with $l \geqslant 2$. Therefore our problem is equivalent to the one posed by Eq. (16) along with the initial conditions

$$
p(z, t=0)=\delta\left(z-z_{0}\right),\left.\frac{\partial p(z, t)}{\partial t}\right|_{t=0}=-c \beta_{1}^{0} \delta^{\prime}\left(z-z_{0}\right) .
$$

Although Eq. (16) is the exact differential equation for the probability density of photons into the medium, it turns out to be very difficult to solve, even approximately, in the time domain. However, as we will shortly see, we can easily get sensible approximations for its time-Laplace transform. Therefore, Eq. (16) seems to be a convenient starting point for continuous-wave experiments (CWE's) where solutions appear in a natural way in the Laplace domain [9]. Indeed, in this type of experiment a continuous source of photons leads the system, after a transient period, to a steady state characterized by the Laplace transform of $p(\boldsymbol{r}, t)$. If the system is absorbent with an absorption rate $\mu_{a}$, then we have to replace, in the Laplace transform $\hat{p}(\boldsymbol{r}, s)$, the variable $s$ by $\mu_{a}$. The Laplace transform of Eq. (16) reads

$$
\begin{aligned}
& \frac{1}{3} c^{2} \sum_{m=0}^{\infty}(-1)^{m+1} \hat{f}_{m}(s) \frac{\partial^{2(m+1)} \hat{p}(z, s)}{\partial z^{2(m+1)}} \\
& \quad+s\left[s+\mu_{s}(1-g)\right] \hat{p}(z, s) \\
&=\left[s+\mu_{s}(1-g)\right] \delta\left(z-z_{0}\right)-c \beta_{1}^{0} \delta^{\prime}\left(z-z_{0}\right)+\hat{g}(z, s) .
\end{aligned}
$$

Equation (18) is an infinite-order differential equation but with constant coefficients. Therefore we have reduced the problem to finding the roots of its characteristic polynomial, which in this case is

$$
q^{2} \mathcal{F}(i q, s)=\frac{3 s}{c^{2}}\left[s+\mu_{s}(1-g)\right]
$$

where $\mathcal{F}$ is the continued fraction defined in Eq. (9) and the $q$ 's are the roots of the characteristic polynomial. It is straightforward to see that in the case of isotropic scattering, the transcendental equation (19) reduces to

$$
\left(s+\mu_{s}\right) \tanh \left(c q / \mu_{s}\right)=c q .
$$
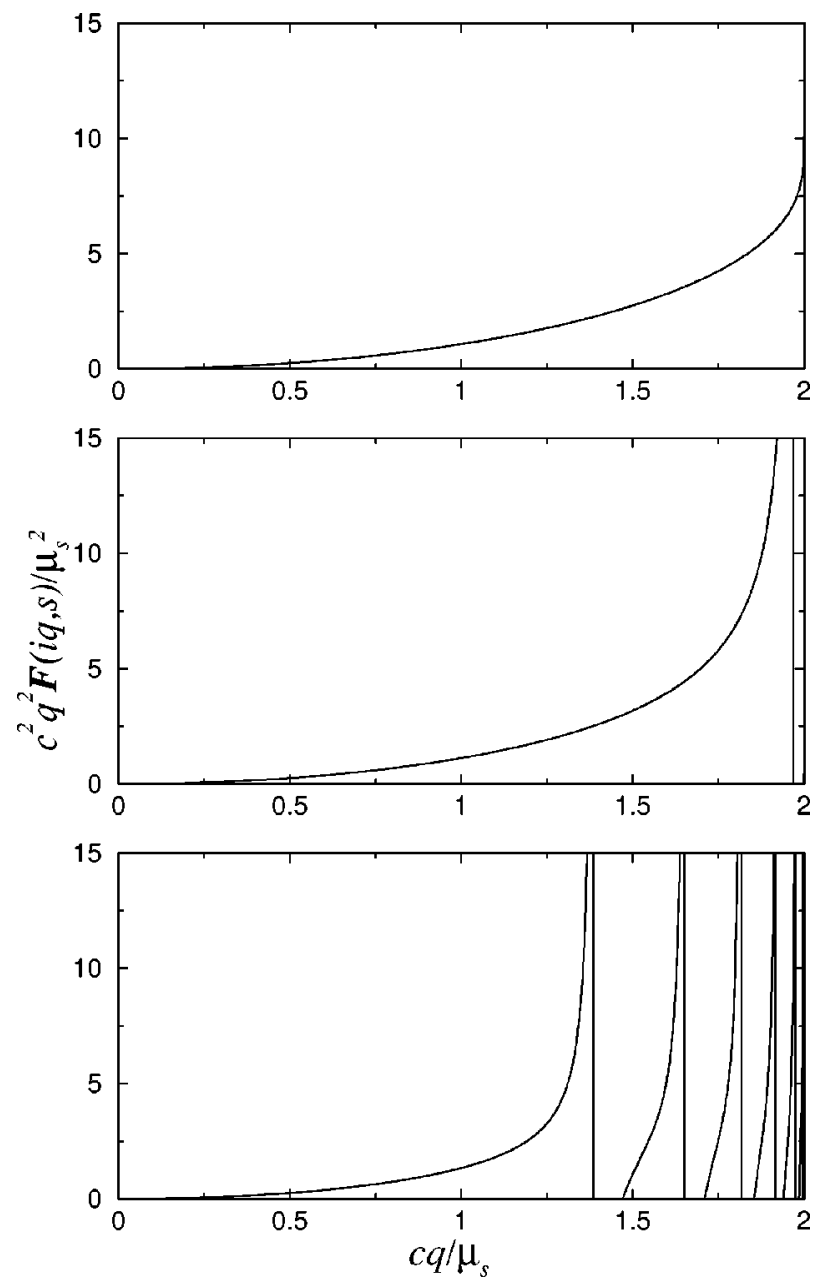

FIG. 1. Numerical solution of the function $q^{2} \mathcal{F}\left(i q, s\right.$ ) (in $\mu_{s}^{2} / c^{2}$ units) as a function of $q$ (in $\mu_{s} / c$ units) for $s=\mu_{s}$ for different values of the anisotropy parameter $g$. From top to bottom: $g=0, g$ $=0.5$, and $g=0.9$.

\section{RESULTS}

The analysis we have done so far is completely general and exact. In order to proceed further with our development, we first need to specify a particular phase function and then obtain some results out of our exact solution that can be tested in practice. We will do it in two steps.

\section{A. The Henyey-Greenstein phase function and the effective absorption}

Unfortunately, it is not possible to obtain the exact solution of the transcendental equation (19), and we have to select a special form for the phase function. We choose the Henyey-Greenstein phase function, which has been used extensively in both numerical analyses and simulations [12]. In this case, the coefficients $\beta_{l}$ have the simple form

$$
\beta_{l}=\beta_{1}^{l}=\langle\cos \theta\rangle^{l} \text {. }
$$

Nevertheless, even with this choice, it is not possible to get a closed analytical expression for the solution of the transcendental equation (19), but the numerical analysis becomes quite simple. In Fig. 1, we show the shape of the function $q^{2} \mathcal{F}(i q, s)$, appearing on the left-hand side of Eq. (19), for 
different values of $g=\langle\cos \theta\rangle$ and for real values of $q$. The first feature we can see is that $q^{2} \mathcal{F}(i q, s)$ is a symmetrical function of $q$ defined in the interval $\left(-s-\mu_{s}, s+\mu_{s}\right)$. This reflects the fact that photons move through the medium at a finite speed and can be directly derived from the structure of the continued fraction $\mathcal{F}$. On the other hand, let $\left\{q_{n}(s)\right\}$ be the set of different roots of Eq. (19). Note that due to the symmetry of $q^{2} \mathcal{F}(i q, s), \pm q_{n}(s)$ are both solutions of Eq. (19). Then for $g=0$, the set of solutions contains only one real root, while the numerical analysis also shows that for an arbitrary value of $g$ the set contains an infinite number of roots whose limit value is

$$
\lim _{n \rightarrow \infty} q_{n}(s)=\frac{1}{c}\left(s+\mu_{s}\right) .
$$

This means that $q(s)=\left(s+\mu_{s}\right) / c$ is an accumulation point of the roots that will lead to an irregular solution associated with ballistic photons. However, this irregular solution contains terms of the order $\exp \left[-\left(s+\mu_{s}\right) z / c\right]$ that will become negligible for $z \gg c\left(s+\mu_{s}\right)^{-1}$. We will focus our interest on the first of these roots, $q_{1}(s)$, because the long-distance behavior of the probability density in the steady state for the CWE is an exponential of rate $q_{1}(s)$. Therefore if we suppose that the system is absorbent with an absorption rate $\mu_{a}$, then the value $c q_{1}\left(s=\mu_{a}\right)$ is exactly the effective absorption rate $\mu_{a}^{*}$; that is,

$$
\mu_{a}^{*}=c q_{1}\left(s=\mu_{a}\right) .
$$

We can see from Eq. (19) that when the absorption is low, the effective rate behaves as

$$
\mu_{a}^{*} \simeq \sqrt{3 \mu_{a} \mu_{s}(1-g)} \quad\left(\mu_{a} \rightarrow 0^{+}\right),
$$

corresponding to the diffusive limit. Moreover, for a strongly absorbing medium we have

$$
\mu_{a}^{*} \simeq \mu_{a} \quad\left(\mu_{a} \rightarrow \infty\right),
$$

corresponding to the ballistic limit. In Fig. 2, we plot this effective absorbing rate for different values of $g$ and the corresponding ones to the diffusion approximation. Observe that the difference between both the exact one and the diffusive one increases when $g$ increases.

\section{B. Some approximations}

In Sec. II preceding section, we obtained the exact differential equation for $\hat{p}(z, s)$ [cf. Eq. (18)]. Nevertheless, this equation is not very useful because we cannot exactly solve an infinite-order differential equation. Therefore we need to do approximations if we want to obtain more information about the process. Since the long-distance behavior in the steady state is governed by the first root $q_{1}(s)$ of Eq. (19), we first approximate the solution by

$$
\hat{p}(z, s) \simeq\left\{\begin{array}{cc}
A e^{q_{1}(s) z}, & z<0 \\
B e^{-q_{1}(s) z}, & z>0 .
\end{array}\right.
$$

The two constants $A$ and $B$ will be determined by the normalization of $\hat{p}(z, s)$ and by the fact that Eq. (20) must re-

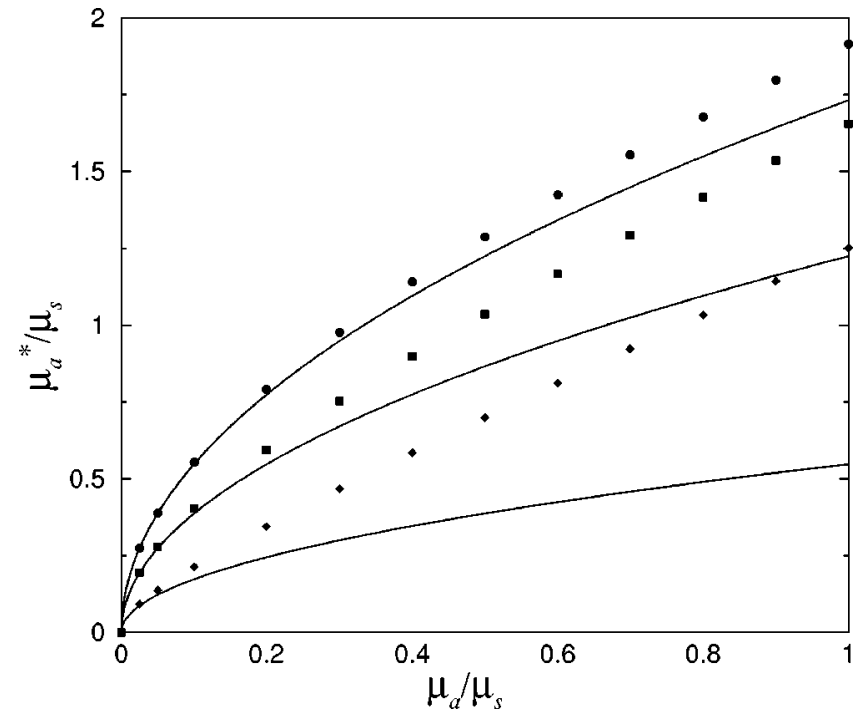

FIG. 2. The effective absorption parameter $\mu_{a}^{*}$ in terms of the absorption parameter $\mu_{a}$ (both $\mu_{a}$ and $\mu_{a}^{*}$ are in $\mu_{s}$ units). Solid lines correspond to the diffusion approximation and symbols correspond to numerical solutions of Eq. (19) for different values of $g: g=0$ (circles), $g=0.5$ (squares), $g=0.9$ (diamonds).

produce the first moment given by Eq. (12). Under these conditions, the differential equation satisfying Eq. (20) reads

$$
\begin{aligned}
& \int_{0}^{t} \alpha(t-\tau)\left[\frac{\partial^{2} p(z, \tau)}{\partial \tau^{2}}+\mu_{s}(1-g) \frac{\partial p(z, \tau)}{\partial \tau}\right] d \tau \\
& =c^{2} \frac{\partial^{2} p(z, t)}{\partial z^{2}}
\end{aligned}
$$

where

$$
\hat{\alpha}(s)=\frac{c^{2} q_{1}^{2}(s)}{s\left[s+\mu_{s}(1-g)\right]},
$$

and the initial conditions are given by Eq. (17). Note that Eq. (21) is a nonlocal telegrapher's equation and it reproduces correctly both the diffusion and the ballistic limit. In addition, Eq. (21) shows the long-distance behavior observed in the CWE. Indeed, when $t \rightarrow 0$ one can easily see from Eq. (22) that $\alpha(t) \sim \delta(t)$, and Eq. (21) reduces to the ordinary telegrapher's equation with a photon speed given by $c$. On the other hand, when $t \rightarrow \infty$ we have $\alpha(t) \sim 3 \delta(t)$, and the telegrapher's equation obtained from Eq. (21) asymptotically results in the following diffusion equation:

$$
\frac{\partial p(z, t)}{\partial t}=\frac{c^{2}}{3 \mu_{s}(1-g)} \frac{\partial^{2} p(z, t)}{\partial z^{2}}
$$

which gives us the correct diffusion coefficient.

The next-order approximation corresponds to taking the first two roots of Eq. (19). In such a case, the solution reads

$$
\hat{p}(z, s) \simeq\left\{\begin{array}{cc}
A_{1} e^{q_{1}(s) z}+A_{2} e^{q_{2}(s) z}, & z<0 \\
B_{1} e^{-q_{1}(s) z}+B_{2} e^{-q_{2}(s) z}, & z>0 .
\end{array}\right.
$$




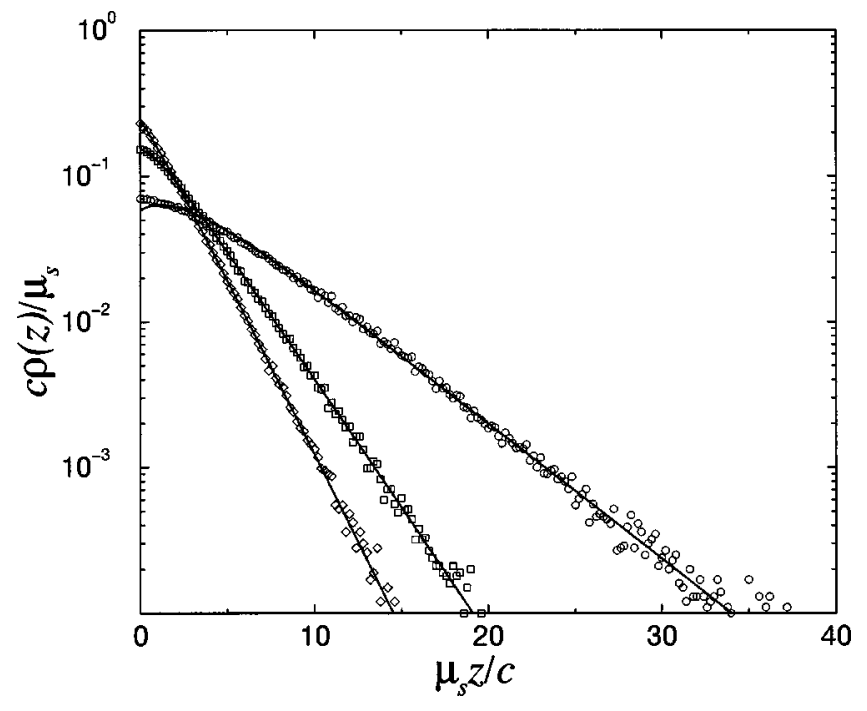

FIG. 3. Normalized concentration profile in the steady state $\rho(z)$ (in $\mu_{s} / c$ units) for $\mu_{a}=0.1 \mu_{s}$. Position $z$ is plotted in $c / \mu_{s}$ units. Solid lines correspond to the numerical solution of Eq. (24) and symbols are simulation results of the continuous time random walk using the Henyey-Greenstein phase function. Photons start moving along the $z$ axis in both directions with equal probability. $g=0$ (diamonds), $g=0.5$ (squares), $g=0.9$ (circles).

As before, the four constants $A_{1}, A_{2}, B_{1}$, and $B_{2}$ must be determined under the conditions that Eq. (23) correctly reproduce the first three moments given by Eqs. (12)-(14) and that it be properly normalized. All of this results in the following differential equation:

$$
\begin{aligned}
& \left(\frac{d^{2}}{d z^{2}}-q_{1}^{2}(s)\right)\left(\frac{d^{2}}{d z^{2}}-q_{2}^{2}(s)\right) \hat{p}(z, s) \\
& =a(s) \delta(z)+b(s) \delta^{\prime}(z)+c(s) \delta^{\prime \prime}(z)+d(s) \delta^{\prime \prime \prime}(z),
\end{aligned}
$$

where

$$
\begin{aligned}
a(s)= & \frac{q_{1}^{2}(s) q_{2}^{2}(s)}{s}, \quad b(s)=-\frac{c \beta_{1}^{0} q_{1}^{2}(s) q_{2}^{2}(s)}{s s_{1}} \\
c(s)= & \frac{c^{2} q_{1}^{2}(s) q_{2}^{2}(s)}{3 s s_{1}}\left(\frac{1}{s}+\frac{2 \beta_{2}^{0}}{s_{2}}\right)-\frac{q_{1}^{2}(s)+q_{2}^{2}(s)}{s} \\
d(s)= & -\frac{c^{3} q_{1}^{2}(s) q_{2}^{2}(s)}{3 s s_{1}}\left(\frac{\beta_{1}^{0}}{s s_{1}}+\frac{4 \beta_{1}^{0}}{5 s_{1} s_{2}}+\frac{6 \beta_{3}^{0}}{5 s_{2} s_{3}}\right) \\
& +\frac{c \beta_{1}^{0}\left[q_{1}^{2}(s)+q_{2}^{2}(s)\right]}{s s_{1}}
\end{aligned}
$$

Again, one can show that Eq. (24) has the right ballistic and diffusive limits. Therefore it seems to be quite a good approximation for the transport process. In order to test this approximation, we have done simulations of the normalized concentration profile in the steady state $\rho(z)$, assuming that there is a continuous source of photons at the origin, and that the medium is absorbent with a rate $\mu_{a}$. We assume that photons start moving along the $z$ axis with equal probability

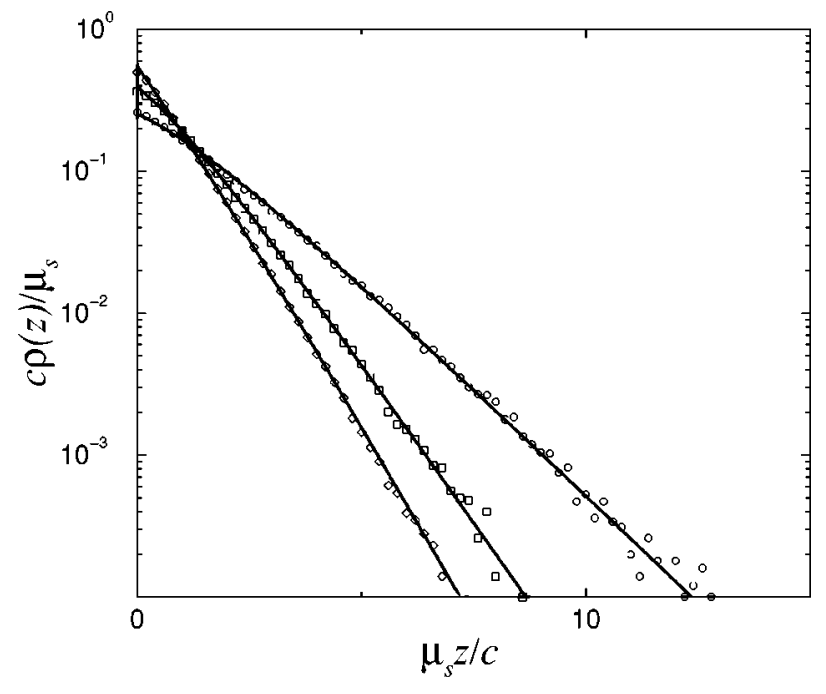

FIG. 4. The same as in Fig. 3, with $\mu_{a}=\mu_{s} / 2$.

for both directions. This last condition corresponds to the choices $\beta_{1}^{0}=\beta_{3}^{0}=0$ and $\beta_{2}^{0}=1$. We also use the HenyeyGreenstein phase function. The normalized concentration profile in the steady state is precisely the normalized solution of Eq. (24), i.e.,

$$
\rho(z)=\left.\hat{s}(z, s)\right|_{s=\mu_{a}},
$$

so we can test the validity of this second-order approximation by (numerically) solving Eq. (24) with the initial conditions explained above. In Figs. 3-5, we plot the concentration profile (28) using the solution of Eq. (24) along with simulations for different values of $g$ and $\mu_{a}$. As we can see in these figures, the agreement between simulations and the solution of Eq. (24) is very good for all values of the parameters, even anisotropic scattering, except for distances less than one length of the scattering mean-free path. This is consistent with the fact that we have neglected the contribution of the roots of Eq. (19) that are greater than $q_{2}(s)$.

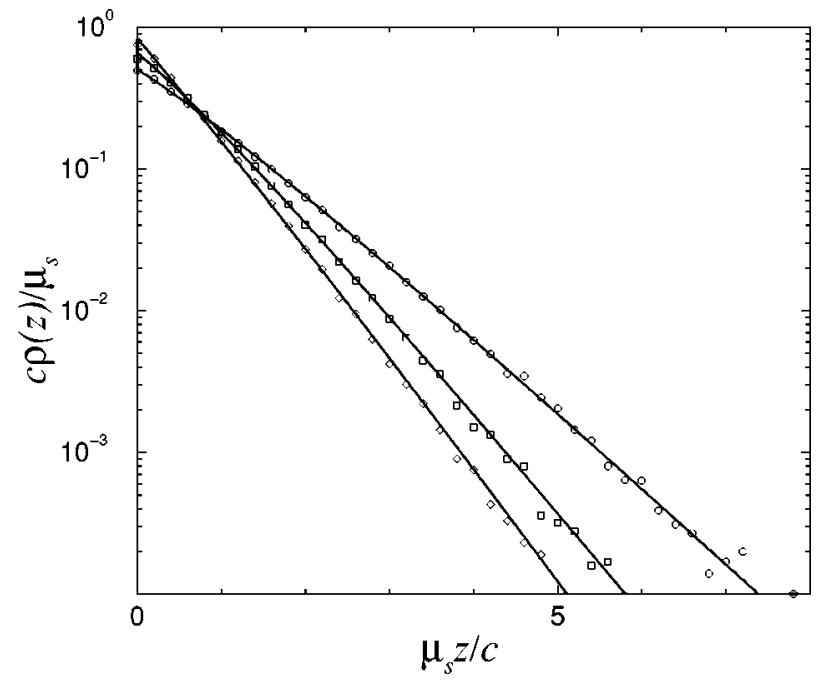

FIG. 5. The same as in Fig. 3, with $\mu_{a}=\mu_{s}$. 


\section{CONCLUSIONS}

In this paper, we have studied the problem of photon propagation through a disordered medium by means of the transport equation for the problem. We have set a successive approximation solution scheme for obtaining the marginal probability density function of photons inside an infinite medium. We have also obtained, in terms of a transcendental equation, the effective absorption parameter, which turns out to be different from that which the diffusion theory provides.

The approximation scheme can be used to solve more realistic problems, such as transport through slabs. A comparison of the theoretical predictions of our procedure with simulations shows that it is a fairly good description of the transport process for all ranges of parameters. In fact, know- ing the differential equation is better adapted to the problem is the first step toward a more complete description of transport in any real problem. In order to accomplish this objective, we need to know the appropriate boundary conditions for the problem. This is not an easy task, and it is presently under investigation.

\section{ACKNOWLEDGMENTS}

We gratefully thank Dr. George H. Weiss for his valuable comments and careful reading of the manuscript. This work has been supported in part by Dirección General de Investigación Científica y Técnica under Contract No. PB96-0188 and Project No. HB119-0104, and by Generalitat de Catalunya under Contract No. 1998 SGR-00015.
[1] B.B. Das, Feng Liu, and R.R. Alfano, Rep. Prog. Phys. 60, 227 (1997).

[2] Optical Tomography, Photon Migration, and Spectroscopy of Tissue and Model Media, edited by B. Chance and R.R. Alfano, SPIE Proceedings No. 2389 (SPIE, Bellingham, 1995); see bibliography in Trends in Optics and Photonics, edited by R. R. Alfano and J. G. Fujimoto (Optical Society of America, Washington, D.C., 1996), Vol 2.

[3] Laser-Doppler Blood Flowmetry, edited by A. P. Shepherd and P. A. Oberg (Luwer, Boston, 1990).

[4] G.H. Weiss, Aspects and Applications of the Random Walk (North-Holland, Amsterdam, 1994).

[5] A.H. Gandjbakhche, R.F. Bonner, and R. Nossal, J. Stat. Phys. 69, 35 (1992).

[6] A.H. Gandjbakhche, R. Nossal, and R.F. Bonner, Appl. Opt. 32, 504 (1993).
[7] A. Ishimaru, Wave Propagation and Scattering in Random Media (Academic, New York, 1978), Vol. 1.; Appl. Opt. 28, 2210 (1989).

[8] D.J. Durian and J. Rudnick, J. Opt. Soc. Am. A 14, 235 (1997).

[9] M. Boguñá, J.M. Porrà, and J. Masoliver, Phys. Rev. E 58, 6992 (1998); 59, 6517 (1999).

[10] J.J. Duderstadt and W. R. Martin, Transport Theory (Wiley, New York, 1979).

[11] J. Masoliver, J.M. Porrà, and G.H. Weiss, Physica A 193, 469 (1992).

[12] L.C. Henyey and J.L. Greenstein, Astrophys. J. 93, 70 (1941).

[13] Handbook of Mathematical Functions, edited by M. Abramowitz and I. A. Stegun (Wiley, New York, 1967).

[14] I. Claes and C. Van den Broeck, J. Stat. Phys. 49, 383 (1987). 